\title{
Adaptación y aplicación del cuestionario CLASS (Colorado Learning Attitudes about Science Survey) para la valoración de actitudes y creencias científicas en alumnos de enseñanza secundaria y universitaria
}

\author{
Juan Argüelles, Carmen Perillán, Paula Núñez
}

Introducción. La evaluación de conocimientos y habilidades ha sido objeto de estudio durante muchos años; sin embargo, la valoración de las actitudes requiere un abordaje diferente.

Objetivo. Utilizar una herramienta metodológica sencilla (cuestionario CLASS-Bio) que permita comparar la evolución de las percepciones acerca de la disciplina de biología y fisiología de los estudiantes noveles, comparándola con las de estudiantes de cursos superiores, con el fin de poder analizar la influencia de la docencia sobre estas percepciones y actitudes.

Sujetos y métodos. Los participantes fueron 288 estudiantes, pertenecientes a dos grupos: un grupo de bachillerato (41 alumnos de primero y 59 de segundo curso) y un grupo de universitarios de primer curso (92 alumnos de enfermería, 53 de fisioterapia y 25 de logopedia). La puntuación favorable representa el porcentaje en que el estudiante contesta como experto.

Resultados. Los resultados fueron más próximos a los obtenidos por el grupo de expertos en segundo curso de bachillerato, con un desplazamiento hacia la mejoría, comparado con los obtenidos en el curso anterior. Las respuestas de grupos de universitarios de enfermería y fisioterapia arrojaron resultados similares, evolucionando hacia unas percepciones más próximas a las de los expertos, y al mismo tiempo bastantes diferentes a las obtenidos con los alumnos de logopedia.

Conclusiones. La adaptación al castellano del cuestionario CLASS-Bio puede utilizarse como herramienta metodológica válida para valorar el proceso continuo de enseñanza-aprendizaje y evaluar la influencia de la docencia sobre las creencias y actitudes de los estudiantes.

Palabras clave. Biología. CLASS. Cuestionario enseñanza-aprendizaje. Fisiología.

Adaptating and implementing CLASS questionnaire (Colorado Learning Attitudes about Science Survey) for assessment of scientific attitudes and beliefs in secondary school and university students

Introduction. Over the last years, evaluation of knowledge and attitudes in biology education has received a strong call to improve.

Aim. To use an easy methodological tool (CLASS-Bio questionnaire) that allows measuring novice-to-expert-like perceptions evolution about Biology and/or Physiology disciplines. In this way, we will be able to analyze the influence over the perceptions and attitudes.

Subjects and methods. The participants were 288 students distributed in two groups: a group of high school students (41 of senior secondary school, 1st course; and 59 of senior secondary school, 2nd course), and another one of undergraduate students (1st year): 92 of Nursing Degree, 53 of Physiotherapy Degree and 25 of Speech Therapist Degree. The favorable rating is quantified by comparing students' responses with a consensus expert response.

Results. The 2nd senior secondary school students' results were more similar to the expert responses than the 1st senior secondary school students; this displacement or shifts indicated an improvement at the 2nd year in the high school. Responses found in the groups of Nursing and Physiotherapy students were similar, evolving closer to the expert perceptions, and quite different compared with the Speech Therapist students responses. It is gratifying to consider how health biosciences students observe these basic subjects more connected to the real world.

Conclusions. Spanish adaptation of CLASS-Bio questionnaire could be used as an accessible methodological tool in order to analyze the continuous evolution of teaching and learning process at different academic stages.

Key words. Biology. CLASS. Physiology. Teaching-learning questionnaire.
Área de Fisiología. Departamento de Biología Funcional. Facultad de Medicina y Ciencias de la Salud. Universidad de Oviedo. Oviedo, Asturias, España.

Correspondencia:

Dr. Juan Argüelles Luis. Área de Fisiología. Departamento de Biología Funcional. Facultad de Medicina y Ciencias de la Salud. Universidad de Oviedo. Julián Clavería, 6. E-33006 Oviedo (Asturias).

Fax:

+34985103534.

E-mail:

jal@uniovi.es

Financiación:

Esta investigación ha recibido el apoyo de la Universidad de Oviedo a través de la Convocatoria de Proyectos de Innovación Docente 2012, código proyecto: PINN-12-061 (Vicerrectorado de Informática).

Nota:

El cuestionario CLASS-Bio está accesible online y en formato PDF en la página web de CLASS: http://www.colorado.edu/sei/ class/CLASS-Bio.html. Las tablas de puntuación en formato Excel también se hallan disponibles en la misma página.

Conflicto de intereses: No declarado.

Competing interests: None declared.

(c) 2015 FEM 


\section{Introducción}

Uno de los objetivos de la implantación del Espacio Europeo de Educación Superior es la adquisición por parte de los estudiantes de competencias, combinación dinámica de conocimientos, habilidades y actitudes, que permitan llevar a cabo una tarea determinada, y considerar que una respuesta competente a un problema profesional es el resultado de esta combinación. La evaluación de conocimientos y habilidades ha sido objeto de estudio durante muchos años, pero la valoración de las actitudes requiere un abordaje diferente.

La investigación en la didáctica de las disciplinas científicas está recibiendo un fuerte impulso tanto en nuestro país como en el ámbito mundial durante las últimas décadas [1-5]. En esta línea, los investigadores en didáctica de las ciencias han identificado una serie de creencias o de actitudes en los estudiantes, a las que van dando forma y moldeando con su experiencia en el aula [6-9]. Tomando como base los estudios acerca de este conjunto de actitudes y creencias en los estudiantes, se han desarrollado diversos instrumentos para sondearlas y analizar su relación con el proceso de aprendizaje [10].

Una forma clásica de analizar el aprendizaje es utilizando comparaciones del tipo experto (profesor)-aprendiz (estudiante). Esta visión supone comenzar describiendo los conocimientos o la forma de razonar del experto o profesor, de tal forma que la enseñanza se dirija a ayudar a los estudiantes a que cambien su estado cognitivo, inicialmente más de tipo aprendiz, hacia un estado más de tipo experto. Así, las descripciones adicionales de lo que saben los estudiantes y de cómo razonan resultarán sumamente valiosas. Conocer en qué difieren los esquemas mentales de los estudiantes respecto de los profesores permitirá a las técnicas de enseñanza ser mucho más precisas y específicas. Esta aproximación del aprendizaje no asume que la experiencia se pueda conseguir con una sola asignatura, ni siquiera al finalizar un curso académico, más bien se trata de un proceso continuo de transformación y de refinamiento.

La didáctica de las ciencias, y más concretamemente de la física, ha utilizado esta técnica de análisis del proceso de enseñanza-aprendizaje. Gran parte de la investigación en este campo se ha centrado en describir cómo y en qué piensan los estudiantes, incluyendo las concepciones acerca de las ideas de la física y su habilidad a la hora de resolver problemas $[7,8]$.

Un paso importante para analizar el proceso supone la creación de sistemas de evaluación capaces de medir si los cambios curriculares y pedagógicos en el aula están teniendo éxito, tanto para mejorar el aprendizaje como en el avance y transición de las actitudes de los estudiantes hacia un pensamiento más de tipo experto $[5,11,12]$.

El cuestionario CLASS (Colorado Learning Attitudes about Science Survey) formó parte inicialmente de los proyectos Phet (Physics Education Technology) y PER@C (Physics Education Research Group at Colorado), desarrollados originalmente en la Universidad de Colorado (Boulder, Estados Unidos). Como curiosidad, cabe destacar que el premio Nobel de Física del año 2001, Carl E. Wieman, formó parte del grupo que elaboró el cuestionario en la citada universidad. El cuestionario CLASS, basándose en las encuestas existentes sobre actitudes y creencias [1315], ha demostrado su importancia en la práctica educativa [16,17]. Metodológicamente se trata de un cuestionario diseñado para poder ser respondido por el alumno en unos 10 minutos, y construido de tal forma que las preguntas sean lo más claras y concisas posibles con el fin de poder adaptarse fácilmente para su uso en una amplia variedad de cursos.

La variante del cuestionario CLASS-Bio [18], utilizada en el presente estudio, se desarrolló a partir del CLASS y ha ayudado a lograr las metas anteriores y permitido comparar las percepciones de los estudiantes noveles, con distintos niveles educativos, en concreto acerca de la disciplina de la biología, con las de los estudiantes más avanzados, de cursos superiores.

\section{Sujetos y métodos}

El estudio se realizó en un total de 288 estudiantes de centros de enseñanza de Oviedo, pertenecientes a dos grupos. Uno de estudiantes de bachillerato, con la siguiente distribución muestral: 41 estudiantes de primer curso $(15,1 \%)$ y 59 de segundo curso $(21,8 \%)$. El segundo grupo corresponde a estudiantes de primer curso de la Universidad de Oviedo: 92 alumnos del grado de enfermería (34,0\%), 53 del grado de fisioterapia $(19,6 \%)$ y 25 del grado de logopedia $(9,2 \%)$. La participación fue voluntaria y se garantizó siempre la confidencialidad y anonimato de los datos proporcionados.

El cuestionario utilizado fue una traducción al castellano de la versión inglesa del CLASS-Bio [18], desarrollado para comparar las diferencias entre las percepciones de los estudiantes 'aprendices' y de 'expertos' sobre el contenido y la estructura de una disciplina específica, en nuestro caso la biología/fisiología, y sus diferentes enfoques en la resolución 
de problemas. El cuestionario consta de 32 preguntas (Tabla I) en las cuales los estudiantes responden utilizando una escala graduada tipo Likert con cinco puntos de respuestas verbales según su grado de acuerdo, desde 'completamente en desacuerdo' hasta 'completamente de acuerdo'.

Respecto a la secuencia temporal, al tratarse de un cuestionario que valora la concepción inicial de una asignatura y la compara con la misma concepción al finalizar su enseñanza o instrucción, obliga a ajustarse a dichos plazos. Así, en las asignaturas anuales, como fueron las de biología de los centros de enseñanza secundaria, se les pasó a los estudiantes dos veces la misma encuesta, una durante la primera semana de clase (septiembre de 2012) y otra al finalizar el curso (junio de 2013). En las enseñanzas universitarias de grado, en los casos de asignaturas de fisiología cuatrimestrales, se les pasó el cuestionario, esta vez cambiando la palabra 'biología' de todas las cuestiones por la palabra 'fisiología', una vez al principio de la docencia (febrero de 2013) y otra al finalizarla (mayo de 2013). De esta forma, el cuestionario permite hacer las comparaciones pre y postinstrucción.

La puntuación favorable 'global' se cuantifica como el porcentaje medio de ítems a los que el estudiante contesta en sentido favorable, es decir, tal y como lo haría un experto. Semsar et al [18] recogieron las respuestas de 69 expertos de 30 universidades estadounidenses y definieron 'experto' como alguien con título de doctor en cualquier campo de la biología; los resultados mostraron que la concordancia media en todas sus respuestas era superior al $90 \%$.

El cuestionario CLASS-Bio se utiliza también para cuantificar categorías específicas de determinadas creencias y actitudes, analizando ciertos subconjuntos de las preguntas (Tabla II). Se utilizaron siete categorías, originalmente obtenidas por los diseñadores del cuestionario, tras realizar un análisis estadístico con muchas respuestas $(n=627)$ en experimentos piloto, de tipo análisis factorial [18].

Los resultados obtenidos por nuestro equipo se trasladaron a una base de datos, Biology Scoring Sheet (http://www.colorado.edu/sei/class/), y se procesaron con el programa estadístico SPSS v. 21. Se analizaron las frecuencias de respuestas para cada apartado de forma global e individual, y su asociación con el tiempo, preinstrucción frente a postinstrucción. El estudio de las diferencias se realizó mediante un análisis de correlación y una prueba de medias apareadas.

Del análisis estadístico de medias apareadas se han excluido los cuestionarios que no pudieron re-
Tabla I. Cuestionario CLASS-Bio.

1. Escogí biología porque me interesan los mecanismos de la vida

2. Me gusta preguntarme acerca de los procesos biológicos que experimentamos en la vida cotidiana

3. Después de estudiar un tema de biología y con la sensación de que ya lo entiendo, me cuesta responder a preguntas sobre el mismo tema

4. El conocimiento de la biología se compone de muchos temas sin conexión entre ellos

5. Cuando respondo a una pregunta de biología, me resulta difícil exponerlo con mis propias palabras

6. No espero que las reglas fundamentales de la biología me ayuden a entender los mecanismos de la vida

7. Para entender un concepto de biología, a veces pienso en mis experiencias personales e intento relacionarlas con el tema que estamos estudiando

8. Si me quedo bloqueado al responder a una pregunta de biología, por lo general trato de imaginarme otra manera diferente de responderla

9. Quiero estudiar biología porque quiero hacer una contribución a la sociedad

10. Si no sé responder una pregunta en un examen de biología, no hay mucho que yo pueda hace (ilegalmente!) para arreglarlo

11. Si quiero usar la misma técnica para entender dos preguntas de biología, éstas tienen que ser similares

12. Me gusta resolver problemas de biología

13. Es importante que el gobierno avale los nuevos descubrimientos científicos antes de que puedan ser aceptados por la sociedad

14. Estudiar biología ha conseguido cambiar mis ideas de cómo funciona el mundo natural

15. Para aprender biología sólo necesito memorizar una serie de hechos y definiciones

16. Los razonamientos utilizados para comprender la biología pueden ser útiles en mi vida cotidiana

17. Resulta útil estudiar los experimentos básicos que apoyan los conceptos generales de biología

18. Si tuviera un montón de tiempo, me gustaría ir a clase de biología (extracurricular) sólo por diversión

19. La asignatura de biología tiene poca relación con lo que experimento en el mundo real

20. Pienso que resolver una cuestión de biología de distintas maneras puede ayudarme a entenderla mejor

21. Si me quedo atascado en una pregunta de biología, no hay ninguna posibilidad de que la resuelva yo solo

22. Al estudiar un tema de biología, relaciono la información importante que ya sé en lugar de simplemente memorizarla

23. Por lo general, sólo hay una única manera correcta de resolver cada problema de biología

24. Cuando no tengo prisa, me gusta seguir dando vueltas a un problema o ejercicio de biología hasta que lo entiendo

25. Si la biología no fuese directamente relevante o aplicable a la salud humana, no me interesaría

26. Las habilidades matemáticas son importantes para entender la biología

27. Me gusta explicar las cosas que aprendo en biología a mis amigos

28. Usamos esta pregunta para descartar a los que no están leyendo las preguntas. Por favor, seleccione 'completamente de acuerdo' a esta pregunta

29. El público en general no entiende muchas ideas o conceptos que se explican en biología

30. Dedico pocos minutos, antes de buscar la ayuda de alguien, tratando de resolver una pregunta de biología

31. Los principios biológicos están sólo para ser memorizados

32. Para mí, la asignatura de biología trata principalmente sobre el aprendizaje de hechos probados, en lugar de investigar lo desconocido 
Tabla II. Categorías del cuestionario CLASS-Bio.

\begin{tabular}{lc}
\hline Categoría & \multicolumn{1}{c}{ Preguntas } \\
\hline Conexión con el mundo real & $2,12,14,16,17,19$ y 25 \\
\hline Conexiones conceptuales/memorización & $6,8,11,15,19,23,31$ y 32 \\
\hline Entretenimiento & $1,2,9,12,18$ y 27 \\
\hline Capacidad de razonamiento & $8,14,16,17$ y 24 \\
\hline Resolución de problemas: dificultad & $3,5,6,10,11,21$ y 30 \\
\hline Resolución de problemas: esfuerzo & $8,12,20,22,24,27$ y 30 \\
\hline Resolución de problemas: estrategias & $7,8,20$ y 22 \\
\hline
\end{tabular}

cogerse en los dos momentos, al principio de la asignatura y al finalizar ésta.

\section{Resultados}

El cuestionario CLASS-Bio se distribuyó en los siguientes niveles de enseñanza: bachillerato (primer y segundo cursos) y universitaria (grados de logopedia, enfermería y fisioterapia), repartidos como se refleja en la tabla III. Se hicieron 275 encuestas al comenzar el curso, repitiéndose al final del periodo de docencia de las asignaturas de biología para el caso de bachillerato o de fisiología en el caso de los grados universitarios. Se contabilizaron 288 encuestas recogidas al finalizar la instrucción.

La tabla IV muestra el número de respuestas favorables de los estudiantes procedentes de los dos niveles educativos (bachillerato y grado universitario), tanto al principio del periodo docente como al finalizar éste, así como su desplazamiento al finalizar la docencia de la asignatura. Una respuesta de los estudiantes se considera favorable si es similar a la respuesta dada por el grupo de expertos. Por ejemplo, si la respuesta del experto es 'de acuerdo', tanto las respuestas de los estudiantes 'completamente de acuerdo' como 'de acuerdo' se cuantifican como favorables.

Como se puede comprobar, los alumnos de segundo de bachillerato fueron los que alcanzaron valores superiores, más cercanos al grupo de expertos, con una mejoría al finalizar la docencia, lo que supuso un desplazamiento en su porcentaje del 2,6\%, mientras que quienes ofrecieron valores porcentuales menores, tanto pre como postintervención, fueron los alumnos de primero de logopedia.
Los cambios producidos en las actitudes y creencias de los estudiantes a lo largo del curso se pueden analizar comparando las medias de los porcentajes favorables en las siete categorías antes de comenzar el curso, con las medias obtenidas al finalizar.

Como se puede comprobar en la gráfica correspondientes a los estudiantes de primero de bachillerato $(n=39)$, se produjo un desplazamiento negativo generalizado de las siete categorías de la percepción de los estudiantes a lo largo del curso académico (Fig. 1), con excepción de la categoría 'resolución de problemas: dificultad' (del 54,9\% al 61,5\%).

Entre los estudiantes de segundo de bachillerato $(n=31)$, el cambio producido es diferente. Según se refleja en la figura 1, a lo largo del curso, el alumno adquiere unas concepciones más próximas a lo que consideran los expertos en la materia, sobre todo en las categorías 'conexión con el mundo real', 'conexiones conceptuales/memorización', 'entretenimiento' y 'grado de dificultad a la hora de resolver problemas'.

Estas diferencias pueden ser comparadas si analizamos simplemente cómo finalizan los alumnos las asignaturas de biología en cada uno de los cursos (postintervención de primer curso frente a postintervención de segundo curso). Cabe destacar la mejoría en los porcentajes 'de acuerdo' de las siete categorías estudiadas (Fig. 1).

Resultados similares se hallaron al pasar el mismo cuestionario a los alumnos de los grados de enfermería $(n=85)$ y fisioterapia $(n=45)$, donde se sustituyó la palabra 'biología' en todas las cuestiones por el término 'fisiología. Destacaron en ambos grados los cambios producidos en las categorías de 'conexión con el mundo real' y 'grado de dificultad a la hora de resolver problemas' -categorías que consideramos fundamentales en este tipo de estudios de biomedicina- y en 'entretenimiento', moviéndose todas ellas hacia una concepción más de tipo experto (Fig. 2).

Resultados diferentes surgieron entre los estudiantes de logopedia $(n=25)$. Este grupo fue escogido debido a que su preparación y experiencias docentes previas son muy diferentes al resto de enseñanzas de ciencias de la vida (Fig. 2). Se puede observar que las categorías antes citadas, como 'conexión con el mundo real', en lugar de tender hacia un concepto más de tipo experto, siguen una tendencia opuesta, hacia un conocimiento de tipo aprendiz.

De hecho, si se analiza conjuntamente el subgrupo enfermería + fisioterapia y se compara con el de logopedia, al finalizar su formación en fisiología, los resultados obtenidos son muy diferentes (Fig. 2). Se puede observar que los estudiantes de enfermería y fi- 
sioterapia se aproximan mucho más a la concepción de los expertos en casi todas las categorías analizadas.

\section{Discusión}

El cuestionario CLASS-Bio constituye un instrumento epistemológico excepcional, ya que según Semsar et al [18], es capaz de comparar directamente las respuestas obtenidas de los estudiantes con las de un grupo de expertos en la materia y además permite agrupar las cuestiones acerca de las creencias de los estudiantes en categorías creadas a partir de datos previos de otros estudiantes. La validez del cuestionario original se ha demostrado al haberse realizado en diferentes grupos de estudiantes y distintos momentos [19,20]. Partiendo del cuestionario original CLASS, para la disciplina de física se han creado dos variantes de éste: una adaptada a la programación curricular de química y otra para biología [18,21].

Un problema inicial que nos encontramos con las encuestas fue tener distinto número de iniciales que de finales debido a que, por ejemplo, algún centro de enseñanza olvidó recordar a sus alumnos encuestados que pusieran su nombre en la encuesta final: resultó imposible reconocer los cuestionarios y no pudieron incluirse en el estudio. Otro problema fue que hubiese más alumnos presentes en clase el día que se pasó la encuesta final que el día de la encuesta inicial. Por tanto, se excluyeron los datos de los alumnos donde no había tanto encuesta inicial como final.

Sin duda, la forma más simple de analizar nuestros datos consiste en observar la tendencia de las percepciones a lo largo del curso. En contra de lo esperado, en muchos de nuestros grupos y categorías encontramos tendencias hacia actitudes tipo aprendiz tras finalizar la docencia de las asignaturas. Especialmente llamativo es el caso de los estudiantes de primero de bachillerato, que acaban el curso con unas actitudes menos próximas a los expertos que al inicio del curso.

Al contrario, la mejoría en las tendencias de actitud en los alumnos de segundo de bachillerato parece indicar que, en el segundo año, los profesores intentaron aproximar con más éxito la disciplina de la biología hacia el mundo real, llamando la atención sobre todo los resultados de la categoría 'resolución de problemas: dificultad'. Esto se pude interpretar diciendo que al finalizar este segundo año de biología, los alumnos tienen una percepción del grado de dificultad de la asignatura más próxima a lo que opinan los expertos.
Tabla III. Distribución de la muestra por sexo y niveles educativos.

\begin{tabular}{|c|c|c|c|c|c|}
\hline \multirow[b]{2}{*}{ Estudios } & \multirow[b]{2}{*}{ Curso } & \multicolumn{2}{|c|}{$\begin{array}{l}\text { Preinstrucción } \\
\quad(n=275)\end{array}$} & \multicolumn{2}{|c|}{$\begin{array}{l}\text { Postinstrucción } \\
\quad(n=288)\end{array}$} \\
\hline & & $\begin{array}{l}\text { Varones } \\
(n=66)\end{array}$ & $\begin{array}{l}\text { Mujeres } \\
(n=209)\end{array}$ & $\begin{array}{l}\text { Varones } \\
(n=89)\end{array}$ & $\begin{array}{l}\text { Mujeres } \\
(n=199)\end{array}$ \\
\hline Colegio A & 2. ${ }^{\circ}$ bachillerato & 5 & 19 & 0 & 0 \\
\hline \multirow{2}{*}{ Colegio B } & 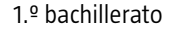 & 3 & 12 & 3 & 12 \\
\hline & 2.. bachillerato & 4 & 19 & 4 & 19 \\
\hline \multirow{2}{*}{ Colegio C } & 1. bachillerato & 18 & 8 & 18 & 8 \\
\hline & 2 bachillerato & 6 & 6 & 6 & 6 \\
\hline Logopedia & Primero & 2 & 28 & 2 & 28 \\
\hline Enfermería & Primero & 10 & 82 & 11 & 87 \\
\hline Fisioterapia & Primero & 18 & 35 & 21 & 39 \\
\hline
\end{tabular}

Tabla IV. Distribución de las respuestas favorables de los estudiantes.

\begin{tabular}{lccc}
\hline & Preinstrucción & Postinstrucción & Desplazamiento \\
\hline 1. o bachillerato $(n=39)$ & $54,0 \%$ & $49,5 \%$ & $-4,5$ \\
\hline 2. o bachillerato $(n=31)$ & $58,4 \%$ & $61,0 \%$ & 2,6 \\
\hline 1. o enfermería $(n=85)$ & $52,7 \%$ & $54,8 \%$ & 2,1 \\
\hline 1. - fisioterapia $(n=45)$ & $52,9 \%$ & $54,2 \%$ & 1,3 \\
\hline 1. o logopedia $(n=25)$ & $50,5 \%$ & $47,0 \%$ & $-3,5$ \\
\hline
\end{tabular}

No podemos olvidar, en el caso de los estudiantes de bachillerato, que los alumnos de segundo curso se encuentran a menudo más seleccionados y presionados por las calificaciones, debido al reducido número de plazas que ofrece la universidad para las carreras biosanitarias. Una posible solución para mejorar los resultados sería implicar más aun a los alumnos en su propio aprendizaje, potenciando la sensación de proximidad entre los problemas biológicos y la vida cotidiana. De esta forma, los alumnos construirán un mayor aprendizaje significativo que permitirá valorar la información adquirida por el estudiante para ser utilizada con facilidad en la resolución de situaciones problemáticas reales.

Con respecto a los alumnos universitarios de primer curso, el resultado inicialmente sorprendente de la tendencia negativa en algunas categorías cons- 
Figura 1. Diferencias en los porcentajes favorables del cuestionario CLASS-Bio del inicio (pre) y del final (post) del curso en estudiantes de primero y segundo de bachillerato.

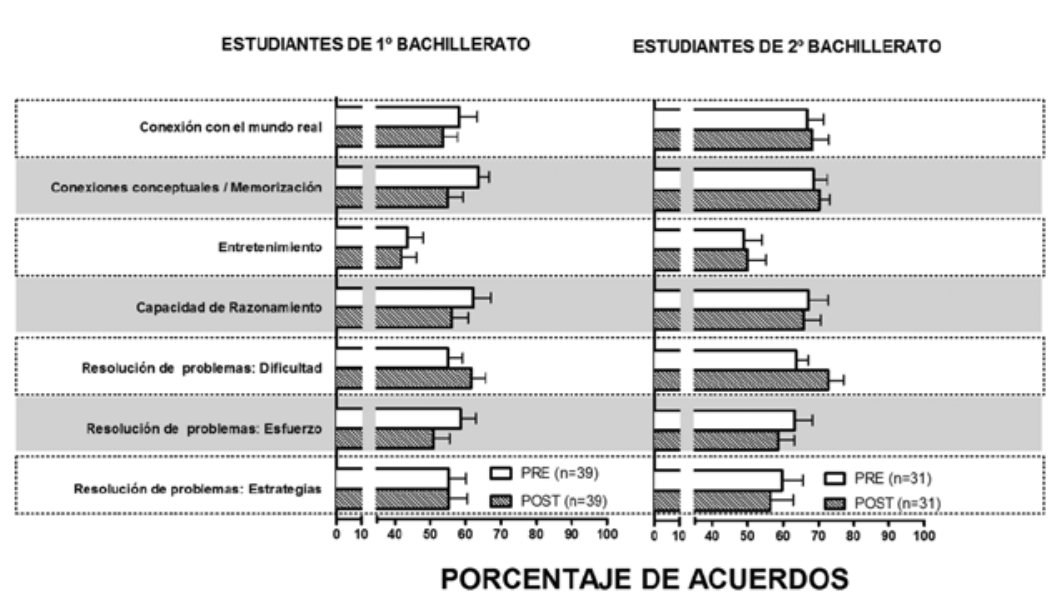

Figura 2. Respuestas favorables (porcentaje de respuestas semejantes a las del 'experto') en los estudiantes de primer curso de los grados de enfermería, fisioterapia y logopedia. ${ }^{*} p<0,05$ (preintervención frente a postintervención).

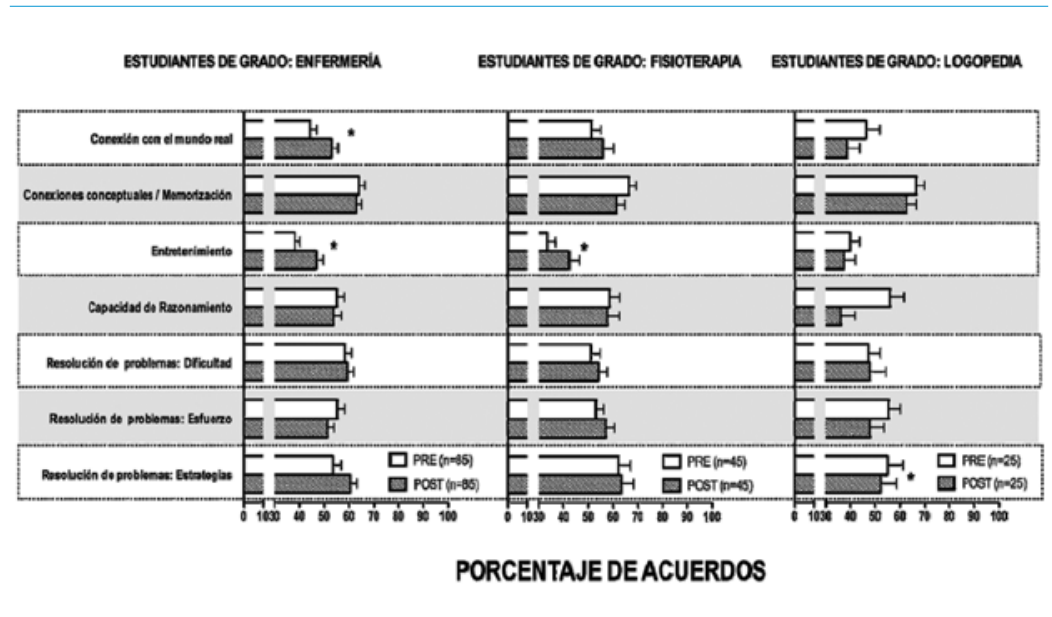

tituye un hallazgo frecuentemente descrito en la bibliografía en las disciplinas de biología, física y química $[18,21,22]$. Algunos autores relacionan este desplazamiento negativo con el poco éxito alcanzado en la docencia a la hora de cambiar las actitudes de los alumnos con los métodos didácticos actuales. Sin duda, nos hace cuestionar qué perseguimos como profesores cuando nos planteamos la docencia de nuestras asignaturas. Probablemente nos centremos más en la adquisición de una serie de conceptos por parte del alumno, sin valorar la posibilidad de un cambio positivo de actitud del alumno hacia nuestra disciplina, o incluso hacia la ciencia en general.

Resulta muy llamativa la concordancia existente en casi todas las categorías entre los estudiantes de los grados de enfermería y fisioterapia. En ambos casos, los mayores desplazamientos hacia la creencia de tipo experto se produjeron en las categorías de 'conexión con el mundo real' y 'entretenimiento', lo cual habla de la apreciación por parte del alumno de la importancia de aplicar los conocimientos que aporta la asignatura de fisiología a su futura tarea profesional, así como de sus factores motivacionales. Por el contrario, entre los alumnos de logopedia sólo se observa un desplazamiento hacia la opinión de tipo experto en la categoría de 'resolución de problemas: dificultad', quizás en parte debido a que estos alumnos provienen de ramas del conocimiento sociales y con una orientación laboral diferente a la de otros estudios más de tipo biosanitario.

Una primera lectura de estos datos señala que, como profesores, deberíamos preocuparnos más de cómo el estudiante adquiere su comprensión conceptual; quizá se precisen aproximaciones pedagógicas diferentes para alcanzar mejor los objetivos perseguidos [16,21]. Dicha metodología permitiría detectar los errores y aprender a manejarlos, poder subsanarlos y continuar de esta forma aprendiendo, y al mismo tiempo, intentar integrar la información adquirida con otras diferentes. El estudiante debería evolucionar hacia un papel más activo, responsable de su saber, y reconocer así su nuevo papel protagonista.

Nuestro estudio puede servir a la hora de plantear acciones de mejora dirigidas hacia la reducción de las deficiencias observadas en la formación de los estudiantes, intentando optimizar la calidad de la enseñanza. Algunas de estas acciones de mejora podrían incluir técnicas de observaciones periódicas de las clases, chequeo de la opinión de los alumnos o estrategias del tipo de aproximación a la realidad, de búsqueda, organización y selección de la información, de descubrimiento, extrapolación y transferencia, de problematización, de procesos de pensamiento creativo divergente y lateral, y de trabajo colaborativo.

Consideramos que este estudio, además, constituye una herramienta metodológica accesible para conocer la evolución año a año del proceso continuo de enseñanza-aprendizaje en la universidad. A través de este tipo de metodología pedagógica resulta más sencillo analizar los progresos o deficiencias que tienen lugar en un determinado ámbito de la formación universitaria en ciencias de la salud, siempre desde el punto de vista del estudiante. 
Bibliografía

1. Jamul D, ed. Beyond Bio101. The transformation of undergraduate biology education: a report from the Howard Hughes Medical Institute. Chevy Chase, MD: Howard Hughes Medical Institute; 2002.

2. National Research Council (NRC). Bio2010: transforming undergraduate education for future research biologists. Washington DC: National Academy Press; 2003.

3. Handelsman J, Ebert-May D, Beichner R, Bruns P, Chang A, DeHaan R, et al. Education. Scientific teaching. Science 2004 304: 521-2.

4. Wood WB. Innovations in teaching undergraduate biology and why we need them. Annu Rev Cell Dev Biol 2009; 25: 51-2.

5. Woodin T, Smith D, Allen D. Transforming undergraduate biology education for all students: an action plan for the twenty-first century. CBE Life Sci Educ 2009; 8: 271-3.

6. Bransford JD, Brown AL, Cocking RR. How people learn. Washington DC: National Academy Press; 2002.

7. Hammer D. Student resources for learning introductory physics Am J Phys 2000; 68: 52-9.

8. Redish EF. Teaching physics with physics suite. Hoboken, NJ John Wiley \& Sons; 2003

9. Seymour E, Hewitt N. Talking about leaving. Boulder, CO: Westview Press; 1997.

10. Seymour E, Zeilik M. Field-tested Learning Assessment Guide (FLAG). URL: http://flaguide.org. [01.03.2014].

11. Garvin-Doxas K, Klymkowsky M, Elrod S. Building, using, and maximizing the impact of concept inventories in the biological sciences: report on a National Science Foundationsponsored conference on the construction of concept inventories in the biological sciences. CBE Life Sci Educ 2007; 6: 277-82

12. Knight JK. Biology concept assessment tools: design and use. Microbiol Aust 2010; 31: 5-8.

13. Redish E, Saul JM, Steinberg, RN. Student expectations in introductory physics. Am J Phys 1998; 66: 212-24.
14. Elby A. Epistemological beliefs assessment for physical science. URL: http://www2.physics.umd.edu/ elby/EBAPS/home.htm. [01.03.2014]

15. Halloun IA. Views about science and physics achievement: the VASS story. In the changing role of physics departments in modern universities: proceedings of the ICUPE, E.F. URL: http://modeling.asu.edu/R\&E/Research.html. [01.03.2014].

16. Perkins KK, Adams WK, Pollock SJ, Finkelstein ND, Wieman CE. Correlating student beliefs with student learning using the Colorado Learning Attitudes about Science Survey. In Physics Education Research Conference. Sacramento, California: AIP; 2004.

17. Perkins KK, Gratny MM, Adams WK, Finkelstein ND, Wieman CE. Towards characterizing the relationship between students' interest in and their beliefs about physics. In Physics Education Research Conference. Sacramento, California: AIP 2006.

18. Semsar K, Knight JK, Birol G, Smith MK. The Colorado Learning Attitudes about Science Survey (CLASS) for use in biology. Life Sci Educ 2011; 10: 268-78.

19. Adams WK, Perkins KK, Podolefsky NS, Dubson M, Finkelstein ND, Wieman CE. New instrument for measuring student beliefs about physics and learning physics: the Colorado Learning Attitudes about Science Survey. Phys Rev ST 2006; 2: 010101.

20. Adams WK, Perkins KK, Dubson M, Finkelstein ND, Wieman CE. The design and validation of the Colorado Learning Attitudes about Science Survey. AIP Conf Proc 2004; 790: 45-8.

21. Barbera J, Adams WK, Wieman CE, Perkins KK. Modifying and validating the Colorado Learning Attitudes about Science Survey for use in chemistry. J Chem Educ 2008; 85: 1435-9.

22. Perkins KK, Barbera J, Adams WK, Wieman CE. Chemistry vs. physics: a comparison of how biology majors view each discipline. PERC Proceedings. Melville NY: AIP Press; 2006. 